Análisis del desempleo y la ocupación después de una política estricta de confinamiento por COVID-19 en Cali

Jhon James Mora 


\section{Análisis del desempleo y la ocupación después de una política estricta de confinamiento por COVID-19 en Cali}

Resumen: Este articulo analiza los efectos de las políticas instauradas de cuarentena, debido al nuevo Coronavirus (COVID-19), sobre la tasa de empleo y desempleo de la ciudad de Cali para el 2020. Debido a que la politica de confinamiento afectó la movilidad de los trabajadores, el principal efecto recayó sobre la generación de empleo de la ciudad. De esta forma, inferir los efectos sobre el empleo se convirtió en uno de los principales retos en la ciudady, con el fin de analizar los impactos del COVID19 sobre el empleo, aqui se propone una metodologia mixta la cual implica, por un lado, información de un comité de expertos en mercado laboral y, por otro lado, el uso de series de tiempo. Los resultados de esta metodología muestran que los pronósticos realizados fueron muy cercanos a lo que se observó en los meses de abril, mayo y junio. Finalmente, en este artículo se analiza el efecto de un shock sobre el mercado laboral de Cali, encontrando que éste duraría alrededor de 18 meses sobre el desempleo y casi un año sobre el empleo.

Palabras clave: empleo; desempleo; informalidad; comité de expertos; series de tiempo; raices unitarias estacionales; Kaplan-Meier.

Clasificación JEL: J11, J23, J64, C32, C41.

\section{Analysis of Unemployment and Employment After a Strict COVID-19 Confinement Policy in Cali}

Abstract: This article discusses the effects of the quarantine policies established due to the new Coronavirus (COVID-19) on the employment and unemployment rates of the city of Cali in 2020. As the policy of confinement affected the mobility of workers, the main effect fell on the generation of employment in the city. Consequently, inferring the effects on employment became one of the main challenges in the city. In order to analyze the impacts of COVID-19 on employment, a mixed methodology is proposed here. On the one hand, it involves information from a committee of labor market experts and, on the other hand, the use of time series. The results of this methodology show that the forecasts made were very close to what was observed in April, May and June. Finally, this article discusses the effect of a shock on Cali's labor market, finding that it would last about 18 months on unemployment and almost a year on employment.

Keywords: employment; unemployment; informality; experts committee; time series; seasonal unit root; Kaplan-Meir.

\section{(cc) BY-NC-SA}

Este artículo y sus anexos se distribuyen por la revista Lecturas de Economía bajo los términos de la Licencia Creative Commons Atribución-NoComercial-CompartirIgual 4.0. https://creativecommons.org/licenses/by-nc-sa/4.0/ 


\section{Analyse du chômage et de l'occupation après une stricte politique de confinement par COVID-19 à Cali}

Résumé: Cet article analyse les effets des politiques de quarantaine mises en place, en raison du nouveau Coronavirus (COVID-19), sur le taux d'emploi et de chômage de la ville de Cali pour l'année 2020. Étant donné que la politique de quarantaine a eu des répercussions sur la mobilité des travailleurs, ceci a eu également des conséquences sur la création d'emplois dans la ville. Déduire les effets sur l'emploi est devenu, ainsi, l'un des principaux défis de la ville. Dans le but d'analyser les impacts de COVID-19 sur l'emploi, on propose une méthodologie mixte qui implique, d'une part, l'information d'un comité d'experts du marché du travail et, d'autre part, l'utilisation de séries chronologiques. Les résultats de cette méthodologie montrent que les prévisions faites étaient très proches de ce qui a été observé au cours des mois d'avril, mai et juin. Enfin, cet article analyse l'effet d'un choc sur le marché du travail de Cali, en constatant qu'il durerait environ 18 mois sur le chômage et presque un an sur l'emploi.

Mots clés: emploi; chômage, informalité; comité d'experts; séries chronologiques; racines unitaires saisonnières; Kaplan-Meier.

Cómo citar / How to cite this item:

Mora, J. J. (2021). El efecto del Coronavirus (COVID-19) sobre el mercado laboral en la ciudad de Cali. Lecturas de Economía, 94, 165-193.

https://doi.org/10.17533/udea.le.n94a342002 


\title{
Análisis del desempleo y la ocupación después de una política estricta de confinamiento por COVID-19 en Cali
}

\author{
Jhon James Mora $\oplus^{\mathrm{a}}$
}

-Introducción. -I. Revisión de literatura. -II. Los datos. -III. COVID-19 y la pérdida de empleos. -IV. El efecto de un shock sobre la tasa de desempleo y ocupación. -Conclusiones. -Agradecimientos. -Referencias.

Primera versión recibida el 09 de mayo de 2020; versión final aceptada el 06 de octubre de 2020

\section{Introducción}

El mercado laboral de la ciudad de Cali ha presentado en los últimos años tasas de desempleo superiores a la tasa de desempleo nacional; entre dos y tres puntos porcentuales ${ }^{1}$. Igual situación se observa para el departamento del Valle. A esta complicada situación, se le suman los efectos de medidas como la cuarentena, implementadas a finales del mes de marzo de 2020, con el fin de reducir los impactos del COVID-19 sobre las vidas de los colombianos.

En la ciudad, se han aplicado estrategias para reducir los efectos sobre el mercado laboral no solo de la pandemia sino también de fenómenos anteriores, como la migración venezolana y la salida de empresas. Sin embargo, el hecho de que exista una gran conmutación laboral con municipios aledaños como Jamundí, Palmira, Candelaria, entre otros, hace que el mercado laboral de Cali sea muy complejo a la hora de su análisis, y que los efectos negativos sobre el empleo en esta ciudad generen, también, impactos negativos sobre estos municipios, y en general sobre el departamento del Valle del Cauca.

Este artículo, busca contribuir al análisis de lo que ha sucedido con la generación de empleo y desempleo en la ciudad de Cali, ante el efecto del

a Jhon James Mora: profesor titular, Escuela de Economía y Finanzas, Facultad de Ciencias Administrativas y Económicas, Universidad Icesi, Colombia. Dirección electrónica: jjmora@icesi.edu.co https://orcid.org/0000-0002-9974-1874

1 De ahora en adelante "p.p." 
Mora: Análisis del desempleo y la ocupación después de una política estricta de confinamiento...

COVID-19, a través del pronóstico de cuál pudo ser la caída en el empleo, utilizando una metodología mixta que combina el uso de información provista por un grupo de expertos y el análisis de series de tiempo.

El aporte principal de este artículo consiste en presentar una novedosa metodología para el análisis del efecto del COVID-19 sobre el empleo. Esta metodología implica mezclar el uso de encuestas a un grupo de expertos sobre el mercado laboral de la ciudad juntamente con el análisis de series de tiempo del empleo con el fin de pronosticar los impactos del shock sobre este en la ciudad de Cali ${ }^{2}$.

La incertidumbre y la inexistencia previa de un fenómeno de tal magnitud en la ciudad, como el shock de la pandemia, llevan a proponer metodologías alternativas para pronosticar cuáles podrían ser los efectos. Los resultados, en uno de los escenarios utilizados, muestran un error menor del $1 \%$ entre las diferencias observadas sobre el empleo generado y la simulación realizada. De esta forma, la contribución es importante en materia de política económica ya que permite a las autoridades municipales tomar acciones con el fin de contrarrestar los efectos negativos sobre el empleo.

Este documento se encuentra estructurado de la siguiente forma. Posterior a la introducción, se presenta la literatura generada durante el 2020 sobre los impactos a nivel mundial y nacional del COVID-19 sobre el empleo. La siguiente sección analiza los datos sobre el empleo en Cali, y otras estadísticas sobre el mercado laboral. Luego, se presenta la metodología para el análisis de los efectos sobre el empleo como causa del COVID-19. La penúltima sección analiza el efecto de un shock sobre las series de tasa de desempleo y empleo. Finalmente, se presentan las conclusiones.

2 Cabe observar que las primeras versiones de este artículo fueron utilizadas como insumo para el análisis de los impactos del COVID-19 sobre el empleo en Cali, en las reuniones de la mesa municipal de empleo de Cali y del Consejo Departamental de Estadística (CODE). De esta forma, ayudó a la política pública de la ciudad y el departamento, al brindar un marco de referencia desde marzo sobre los impactos que el COVID-19 tendría sobre el empleo de la ciudad y sobre el Valle del Cauca dada la importancia del mercado laboral caleño en el mercado laboral departamental. 


\section{Revisión de literatura}

Como es de amplio conocimiento, el primer caso de COVID-19 fue registrado en el mercado de comida de mar de Wuhan (ciudad capital de la provincia Hubei, China) el 8 de diciembre de 2019 (Qiu, et al., 2020).

$\mathrm{Al}$ ser un fenómeno reciente, la literatura con respecto a los efectos de un shock de este tipo sobre el empleo no es muy abundante, no tanto porque no existan artículos relacionados con crisis económicas y desempleo/empleo, sino por las particularidades que implicó el COVID-19, como fue la imposición de medidas de cuarentena y cierre de mercados a nivel mundial.

Como bien lo plantean Angelucci et al. (2020): "A diferencia de recesiones anteriores, la pandemia COVID-19 puede haber causado pérdidas de empleo y salud desproporcionadas para los trabajadores cuyos puestos de trabajo no podrían llevarse a cabo de forma remota" (p. 1).

Alstadsaeter et al. (2020) analizan la magnitud, la dinámica y los aspectos socioeconómicos de los despidos temporales y permanentes provocados por la crisis del COVID-19 en Noruega. Sus resultados muestran que cerca del $90 \%$ de los despidos son temporales. Adicionalmente, el riesgo de despido es mayor para aquella población económicamente vulnerable, es decir, individuos con bajos ingresos, educación, salario por hora y clase social. Finalmente, los autores establecen que el sector público se encuentra ampliamente protegido contra los despidos, mientras que el sector privado se caracteriza por una mayor probabilidad de despido para las mujeres con niños pequeños.

Bartik et al. (2020) analizan los efectos sobre el mercado laboral del programa estadounidense CARES (Coronavirus Aid, Relief, and Economic Security) el cual, además de extender los beneficios del desempleo, proporcionó ayudas a la protección del empleo en pequeñas empresas. Los resultados muestran que el mercado laboral de Estados Unidos presentó una recuperación a mediados de abril y junio, periodo en el que se estabilizó y comenzó a declinar nuevamente debido a los efectos del COVID. Con respecto a las políticas públicas, los autores indican que la velocidad de la 
Mora: Análisis del desempleo y la ocupación después de una política estricta de confinamiento...

recesión determina la importancia de los programas automáticos, destacando las limitaciones de las políticas ad hoc.

Boar y Mongey (2020) también analizan el impacto de "CARES" en Estados Unidos como medida para sobrellevar la crisis de desempleo como resultado de la pandemia. Muestran que solo los trabajadores con un salario previo bajo no estarían dispuestos a regresar a su antiguo trabajo, lo cual representa una proporción de trabajadores relativamente pequeña. Lo anterior, permite a los autores concluir que la probabilidad de que el elevado desempleo presentado entre abril y julio se deba a la instauración de la ley CARES es baja.

Coibion et al. (2020) estudian cómo el mercado laboral estadounidense se ha visto afectado por el COVID-19 a partir de la relación empleopoblación, la tasa de desempleo y la tasa de participación en la fuerza laboral. Los autores encuentran que la tasa de empleo se redujo del $60 \%$ al 52,2\%; específicamente, dicha disminución es superior a la disminución experimentada durante la Gran Recesión (1929). Por otro lado, la tasa de desempleo pasó del 4,2\% al 6,3\% y, por último, la participación en la fuerza laboral disminuyó en aproximadamente 7 p.p.

Dingel y Neiman (2020) determinan la cantidad de trabajos que pueden realizarse desde casa, considerando las medidas de distanciamiento social adoptadas en Estados Unidos para detener la propagación del COVID19. Sus resultados muestran que el $37 \%$ de los trabajos se pueden realizar completamente en casa, con una variación significativa entre ciudades e industrias, y que, por lo general, estos trabajos pagan más que aquellos que no se pueden realizar en casa, representando el $46 \%$ de todos los salarios estadounidenses. Por último, a través de la reorganización de la clasificación de grupos de actividades económicas con la de la Organización Internacional del Trabajo (OIT), encuentran que los hogares de menores ingresos tienen una menor proporción de trabajos que se pueden realizar desde el hogar.

Forsythe et al. (2020) analizan el impacto del COVID-19 en el mercado laboral estadounidense. Para ello, utilizan los datos de puestos vacantes recopilados en tiempo real por Burning Glass Technologies (BGT), los datos de reclamos iniciales del seguro de desempleo (UI, por sus siglas en 
inglés) del departamento de trabajo de Estados Unidos y los datos de empleo de la Oficina de Estadísticas Laborales (BLS, siglas en inglés). Sus resultados muestran que el mercado laboral estadounidense experimentó una disminución general en las ofertas de trabajo, aproximadamente del $40 \%$ para finales de abril, y un aumento en las reclamaciones de seguros en los diferentes estados, sectores y ocupaciones. En cuanto a los sectores económicos, los autores establecen que las mayores pérdidas se produjeron en ocio y hostelería, y comercio minorista no esencial, donde de febrero a abril las ofertas de empleo cayeron por debajo del $50 \%$ y el empleo disminuyó a la mitad para ocio y hostelería, y a un tercio en comercio minorista no esencial.

En Colombia por su parte, se han realizado los siguientes trabajos que relacionan el COVID-19 con el mercado laboral:

Alfaro et al. (2020) cuantifican la exposición económica y a la enfermedad en el trabajo, generada por los cierres, a través de la utilización de datos de la Gran Encuesta Integrada de Hogares 2019 y la matriz insumo-producto del DANE 2015. Los resultados muestran que, en los sectores vulnerables, las personas ocupadas con mayor exposición económica, como resultado de la pandemia, son quienes trabajan en una empresa de 10 empleados o menos, seguido por aquellas con empleo informal. Los autores también encuentran que los principales determinantes de la exposición a la enfermedad en el trabajo, en sectores vulnerables, son aquellos asociados al alto contacto físico, con un $80,3 \%$, seguido del uso del transporte público, para desplazarse al punto de trabajo, con un 30,5\%. Por último, destacan que los sectores con mayor exposición a la enfermedad en el trabajo son las minas y canteras, construcción y transporte de pasajeros.

Botero y Montañez (2020), utilizando un modelo de equilibrio general computable, encuentran que el PIB caería hasta un 3,9\% para el 2020 y los efectos del COVID-19 persistirían hasta el año 2021. De esta forma "las medidas de contención (escenario COVID+CC), que limitan de manera efectiva los contactos interpersonales y distancian socialmente a las personas, agudizan inicialmente los impactos económicos de la crisis, profundizando la caída del PIB en el segundo trimestre al $11,1 \%$ vs una caída del 6,5\% en el escenario COVID. Pero, si son efectivas, deberían permitir una gradual 
Mora: Análisis del desempleo y la ocupación después de una política estricta de confinamiento...

recuperación de la economía, que permitiese cerrar el año con una caída de sólo el 2,2\%, frente al 3,9\% del escenario sin contención” (p. 18).

Bonet et al. (2020) utilizando una modelo de insumo-producto, estiman la pérdida económica que resulta de extraer un grupo de empleados formales e informales de los distintos sectores y entidades territoriales del país. De esta forma, los resultados muestran pérdidas económicas varían entre un $0,5 \%$ y $6,1 \%$ del PIB nacional.

Eslava e Isaacs (2020) determinan la vulnerabilidad del empleo frente a la contingencia del COVID-19 a partir de los sectores que presentan restricción en la movilidad y son inflexibles al trabajo remoto. Los resultados muestran que los sectores más vulnerables corresponden a la construcción, comercio al por mayor y al por menor de productos no básicos, alojamiento y restaurantes, transporte, servicios domésticos, entre otros. Estos sectores presentan un gran número de ocupados y nivel de ingresos. Finalmente, los sectores más vulnerables se encuentran conformados, en mayor medida, por trabajadores informales (no cotizantes a pensión).

Fernández (2020) analiza cómo las medidas de aislamiento implementadas en marzo y abril afectan el mercado laboral colombiano, con base en datos de la GEIH. Los resultados muestran que los trabajadores más afectados por el aislamiento obligatorio están conformados, en su mayoría, por trabajadores independientes y asalariados informales, pertenecientes a sectores no prioritarios y no adaptables. Por otro lado, este tipo de sectores constituye los más afectados, resaltando el comercio, vendedores de productos no alimenticios, hoteles y bares, y otros servicios. De esta forma, los ingresos laborales más afectados corresponden a aquellos de los trabajadores independientes que pertenecen a dichos sectores.

Jaramillo et al. (2020) plantean que los efectos de la medida de aislamiento obligatorio impactan de manera diferencial a los trabajadores formales e informales. Además, discuten las cuestiones legales en torno a la contratación y estabilidad laboral para la protección del trabajador, y lo referente a las licencias no remuneradas y revisiones de contrato, proponiendo un control adecuado y el cumplimiento de los ordenamientos laborales. Igualmente, los autores analizan los efectos de la pandemia sobre los ingresos 
de los hogares vulnerables y aquellos individuos que no cuentan con sistemas de seguridad social.

Morales et al. (2020) analizan cómo la pandemia por COVID-19 y las recientes restricciones sectoriales de movilidad para la mitigación de contagios, afectaron durante el primer cuatrimestre del año el mercado laboral en Colombia. Los resultados indican que las restricciones específicas del sector tuvieron un efecto negativo sobre el empleo. Específicamente, una cuarta parte de la pérdida total de empleo se debe a las restricciones entre febrero y abril. Por otro lado, los autores establecen que las restricciones específicas del sector no tienen efectos significativos sobre el número de horas trabajadas o salarios promedio.

Finalmente, Serna et al. (2020) proponen un índice para medir la vulnerabilidad del empleo frente al COVID-19, denominado Índice de Riesgo a la Pérdida del Empleo. Los resultados muestran que los sectores más afectados serían los que corresponden a construcción, comercio, actividades inmobiliarias, transporte e industria manufacturera. Además, las ciudades que sufrirían mayores impactos en el mercado laboral serian Cúcuta, Cartagena, Medellín, Pereira y Barranquilla. De esta forma, el riesgo de perder el trabajo se estima alrededor del $20 \%$ para quienes no tienen contrato definido, frente al 14,4\% de riesgo de perder el trabajo para la población general en las trece ciudades principales. En cuanto al sitio del empleo, los autores encontraron que aquellos individuos que usan transporte público para dirigirse a su trabajo tienen un riesgo más alto de perder su empleo. Finalmente, los trabajadores que tienen una posición de subordinación suelen presentar mayor vulnerabilidad en el empleo.

\section{Los datos}

La ciudad de Cali se ha caracterizado en los últimos años, no solo por su alta tasa de desempleo (casi 3 p.p. por encima del nivel nacional.), sino también por generar empleo (entre enero y febrero del 2020 creció en casi 5 p.p.).

La Tabla 1, muestra la información para el año 2019, y los datos disponibles para 2020, obtenidos a partir de la encuesta GEIH para Cali: 
Mora: Análisis del desempleo y la ocupación después de una política estricta de confinamiento...

Tabla 1. Indicadores del mercado laboral para Cali-AM

\begin{tabular}{cccccc}
\hline Año & Mes & TD & TO & TI-INS & TI-DANE \\
\hline \multirow{7}{*}{2019} & $12,13 \%$ & $58,31 \%$ & $53,74 \%$ & $45,72 \%$ \\
& Ene & $12,95 \%$ & $58,05 \%$ & $56,31 \%$ & $45,17 \%$ \\
& Mar & $13,53 \%$ & $58,35 \%$ & $55,24 \%$ & $45,76 \%$ \\
& Abr & $13,18 \%$ & $59,15 \%$ & $56,96 \%$ & $46,47 \%$ \\
& May & $13,00 \%$ & $59,42 \%$ & $53,81 \%$ & $45,24 \%$ \\
& Jun & $12,58 \%$ & $59,19 \%$ & $55,77 \%$ & $45,84 \%$ \\
& Jul & $11,35 \%$ & $60,49 \%$ & $55,00 \%$ & $49,54 \%$ \\
& Ago & $11,75 \%$ & $60,47 \%$ & $55,85 \%$ & $46,67 \%$ \\
& Sep & $11,75 \%$ & $60,59 \%$ & $52,44 \%$ & $42,55 \%$ \\
& Oct & $12,47 \%$ & $59,53 \%$ & $55,00 \%$ & $44,09 \%$ \\
& Nov & $12,07 \%$ & $59,51 \%$ & $54,46 \%$ & $45,42 \%$ \\
& Dic & $12,05 \%$ & $60,38 \%$ & $56,67 \%$ & $45,34 \%$ \\
\hline \multirow{2}{*}{2020} & Mes & TD & TO $\%$ & TI-INS $\%$ & TI-DANE \\
\hline & Ene & $15,70 \%$ & $56,94 \%$ & $59,10 \%$ & $48,04 \%$ \\
& Feb & $12,12 \%$ & $60,07 \%$ & $58,03 \%$ & $49,19 \%$ \\
& Mar & $14,23 \%$ & $52,41 \%$ & N.D. & N.D. \\
& Abr & $25,57 \%$ & $38,84 \%$ & N.D. & N.D. \\
& May & $29,78 \%$ & $41,64 \%$ & N.D. & $45,64 \%$ \\
& Jun & $27,54 \%$ & $46,03 \%$ & N.D. & $50,29 \%$ \\
& Jul & $27,49 \%$ & $46,43 \%$ & N.D. & $55,74 \%$ \\
\hline
\end{tabular}

Fuente: elaboración propia a partir de la GEIH-DANE.

En la Tabla 1, la información sobre la tasa de desempleo (TD) y la tasa de ocupación (TO) corresponden al trimestre móvil. La información del 2020 muestra como a medida que se extiende la pandemia (y, por lo tanto, se utilizan medidas como la cuarentena), la tasa de desempleo se incrementa mientras que la tasa de ocupación cae. La diferencia, con respecto a la información mensual, es casi alrededor de 4 p.p. en el caso del desempleo. Es así como, mientras la tasa de desempleo trimestral fue del 23,01 \% en el trimestre marzomayo, la tasa de desempleo del mes de mayo fue del $29,8 \%$ mientras la de junio fue de $27,75 \%$ mostrando una reducción leve. 
Referente a la tasa de informalidad, esta se encuentra entre el $45 \%$ y el $55 \%$ aproximadamente, dependiendo de la forma en la que se mide. La informalidad institucional (TI-INS) (afiliación a la seguridad social y contrato) arroja valores por encima del $50 \%$, mientras que la informalidad medida por del DANE (TI-DANE) arroja valores alrededor del 50\% mensuales ${ }^{3}{ }^{4}$. De esta forma, en media, uno de dos trabajadores en la ciudad se encuentra en condiciones de informalidad laboral en nuestra ciudad.

Sin embargo, la situación de informalidad no es la misma por estrato socioeconómico de la población, tamaño de las empresas o sector económico. Esto se puede observar, a continuación, en las tablas 2, 3 y 4:

Tabla 2. Informalidad por estrato socioeconómico (PRE COVID-19)

\begin{tabular}{lcccc}
\hline Estrato & Inform. & Institucional & Inform. & DANE \\
\hline 1 & 200187 & $27,10 \%$ & 183046 & $29,20 \%$ \\
2 & 229141 & $31,00 \%$ & 187930 & $29,97 \%$ \\
3 & 224761 & $30,40 \%$ & 180028 & $28,71 \%$ \\
4 & 18556 & $2,50 \%$ & 19118 & $3,05 \%$ \\
5 & 24408 & $3,30 \%$ & 15693 & $2,50 \%$ \\
6 & 18444 & $2,50 \%$ & 21204 & $3,38 \%$ \\
$\mathrm{~N} s / \mathrm{Nr}$ & 24110 & $3,30 \%$ & 19942 & $3,18 \%$ \\
\hline Total & 739607 & $100,00 \%$ & 626961 & $100,00 \%$ \\
\hline
\end{tabular}

Fuente: elaboración propia a partir de la GEIH (febrero, 2020).

La Tabla 2 muestra que, alrededor de la mitad de los trabajadores informales se concentra en los estratos 1 y 2 . En el estrato 3 se concentra

3 Ver Mora (2017) para un análisis de la informalidad en Colombia.

4 Los cálculos para los meses de marzo y abril no se pudieron procesar debido a que las encuestas de la GEIH se realizaron telefónicamente, en entrevistas de 20 minutos, lo cual llevó a eliminar las preguntas que permitían el cálculo de la informalidad institucional y estructural. En los meses de mayo y junio se retomaron preguntas que permitían calcular la informalidad del DANE, pero no la institucional. Sin embargo, los datos de informalidad hay que tomarlos con cuidado ya que en el mes de mayo aparentemente se redujo la informalidad tipo DANE en 4 p.p. durante pleno COVID-19.

5 No se puede calcular la información de la informalidad institucional en mayo debido a que la información recopilada por el DANE para ese mes no lo permite. 
Mora: Análisis del desempleo y la ocupación después de una política estricta de confinamiento...

un poco más del $28 \%$ de los trabajadores y en los estratos 4 al 6 se concentra alrededor del $8 \%$ de los trabajadores informales en la ciudad de Cali.

En cuanto a la informalidad institucional por tamaño de la empresa (a partir del número de empleados), se puede observar lo siguiente:

Tabla 3. Informalidad por número de empleados en la empresa

\begin{tabular}{lcccccc}
\hline No. Empleados/Sector & \multicolumn{2}{c}{ Informal } & \multicolumn{2}{c}{ Formal } & \multicolumn{2}{c}{ Total } \\
\hline Trabaja solo & 340401 & $96,01 \%$ & 14137 & $3,987 \%$ & 354538 & $27,8 \%$ \\
2 a 3 personas & 183311 & $91,72 \%$ & 16542 & $8,277 \%$ & 199854 & $15,7 \%$ \\
4 a 5 personas & 85095 & $81,86 \%$ & 18860 & $18,143 \%$ & 103955 & $8,2 \%$ \\
6 a 10 personas & 53057 & $60,09 \%$ & 35236 & $39,908 \%$ & 88293 & $6,9 \%$ \\
11 a 19 personas & 24904 & $44,37 \%$ & 31220 & $55,627 \%$ & 56123 & $4,4 \%$ \\
20 a 30 personas & 18404 & $35,12 \%$ & 33997 & $64,878 \%$ & 52401 & $4,1 \%$ \\
31 a 50 personas & 9980 & $26,61 \%$ & 27530 & $73,394 \%$ & 37510 & $2,9 \%$ \\
51 a 100 personas & 5244 & $15,20 \%$ & 29260 & $84,802 \%$ & 34504 & $2,7 \%$ \\
101 o más personas & 19210 & $5,53 \%$ & 328188 & $94,470 \%$ & 347398 & $27,3 \%$ \\
\hline Total & 739607 & $58,03 \%$ & 534969 & $41,972 \%$ & 1274576 & $100,0 \%$ \\
\hline
\end{tabular}

Fuente: elaboración propia a partir de la GEIH (febrero, 2020).

De acuerdo con la Tabla 3, el $27 \%$ de los trabajadores se encuentra en empresas unipersonales y, de estos, el $96 \%$ no tiene cotización a pensión, y algunos tampoco tienen salud. La Tabla 3 también muestra como a medida que se incrementa el número de trabajadores - empresas más grandes - el porcentaje de informalidad se reduce.

Por último, la cantidad de ocupados e informales laborales no es la misma en todos los sectores, como se puede observar a continuación:

La Tabla 4, indica que, para los dos primeros meses del 2020, el sector que más empleo genera en la ciudad es el comercio; este también es el sector que más trabajadores informales agrupa, tanto institucionales como medidos por el DANE (estructurales). El segundo sector es el de industrias manufactureras y está seguido por hoteles y restaurantes, transporte y actividades inmobiliarias. Estos cinco sectores concentran alrededor del $64 \%$ del empleo y, entre el $69 \%$ y el $70 \%$ de la informalidad, dependiendo del mes. 


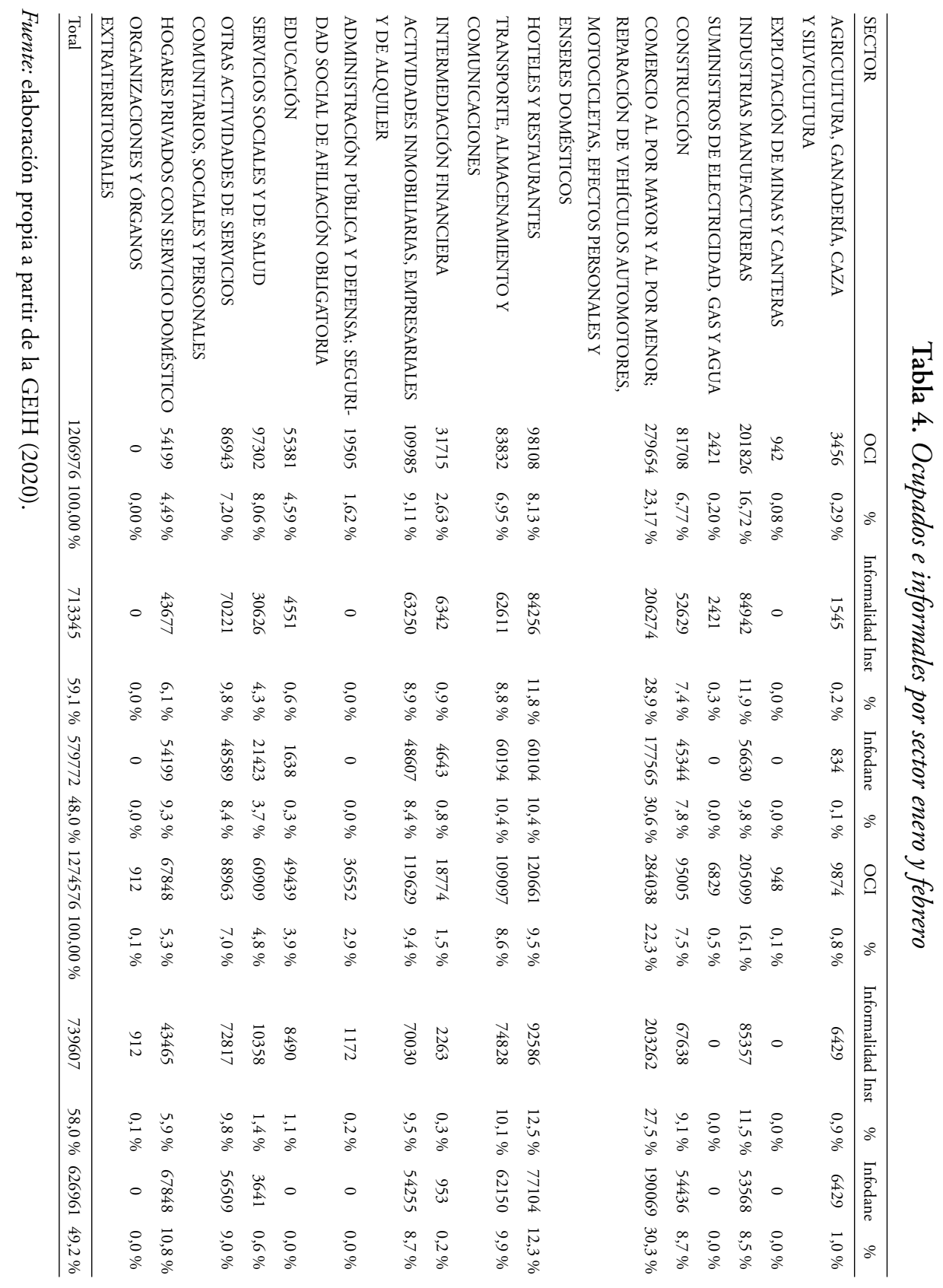


Mora: Análisis del desempleo y la ocupación después de una política estricta de confinamiento...

\section{COVID-19 y la pérdida de empleos}

Sin lugar a duda, esta es una de las preguntas más complejas de responder hoy día. Al momento solo existen algunas consideraciones sectoriales y solo, probablemente, en algún par de meses podremos cuantificar de manera exacta las perdidas en el empleo. Aun así, trataremos de aproximarnos al efecto del COVID-19 sobre la generación de empleo.

Desde el punto de vista metodológico, aproximarse a los efectos es un problema algo complejo. Sin embargo, utilizaremos una metodología mixta con el fin de lograrlo.

Para este propósito, en este artículo se utilizará como aproximación metodológica, la combinación de la información sobre los efectos del COVID-19 sobre la pérdida de empleos, a través de la consulta a expertos y el uso de series de tiempo. Esta combinación nos permite calcular cuál podría ser el empleo en el 2020 y, una vez obtenido éste, se utilizará la información arrojada por los expertos para obtener el total de empleo en un mes determinado como resultado del efecto de la pandemia sobre el empleo de la ciudad.

En primer lugar, con el fin de "agrupar" la información proveniente de la consulta a expertos, se propone un modelo de opinión lineal conjunta (Linear Opinion Pool), el cual se utiliza para combinar las opiniones de expertos (Seaver, 1978; Clemen y Winkler; 2007; Genest y McConway, 1990; Mora y Marulanda, 2018).

Sea $n$ el número de expertos, $f_{i}(\theta)$ la distribución de probabilidad asignada por el experto a la cantidad de interés desconocida $\theta \in \Theta$ y $w_{i}$ la ponderación asignada a cada experto. Entonces la distribución combinada probabilística se puede expresar de la siguiente forma:

$$
T\left(f_{1}, \ldots, f_{n}\right)=\sum_{i=1}^{n} w_{i} f_{i} ; \quad \sum_{i=1}^{n} w_{i}=1 .
$$

Un punto crucial en la ecuación 1 consiste en cómo asignar $w_{i}$, ya que este representa la calidad relativa del experto (Mora y Caicedo, 2018). Por 
otro lado "La asignación de $w$ es subjetiva y podría dar lugar a numerosas interpretaciones (Genest y McConway, 1990). Seaver (1978) encuentra que el desempeño obtenido a partir de ponderaciones diferentes es muy similar y que un procedimiento simple como el de asignar igual peso a cada experto da resultados similares a aquellos en los cuales se utilizaron métodos complejos de agregación. Y, como bien lo plantean Clemen y Winkler (2006), el mensaje en general consiste en que los métodos de agregación simples se desempeñan mejor que los métodos más complejos” (Mora y Caicedo, 2018: 45). Adicionalmente, como concluyen Genest y McConway (1990) "Si el DM no dispone de información suficiente para realizar esta evaluación inicial, podría invocar algún tipo de «principio de razón insuficiente» para asignar a todas las opiniones el mismo peso. En su documento de revisión, Hogarth (1975) informa que la ponderación igual generalmente funciona bien en comparación con otros esquemas que intentan sopesar a los evaluadores de acuerdo con su experiencia” (p. 57).

La información para el cálculo de la pérdida de empleos proviene de una consulta a los expertos tanto del Observatorio Regional del Mercado de Trabajo, ORMET, como del Sistema Municipal de Empleo, SIME, de la Alcaldía de Santiago de Cali, en las dos primeras semanas del mes de abril y que fue respondida por 19 de los miembros, los cuales se reúnen de manera periódica, en el ORMET Valle - SIME, con el fin de analizar el mercado laboral de la ciudad de Cali.

A los expertos, se les pregunto cuál sería el porcentaje de reducción en empleos para cada uno de los sectores clasificados a un dígito. El resultado obtenido, utilizando el modelo de opinión lineal conjunta, se presenta en la Tabla 5.

La Tabla 5, muestra como los miembros del ORMET consideran que la mayor reducción en el empleo se presentará en el sector de otras actividades de servicios comunitarios mientras que el sector con menor reducción sería el de servicios sociales y de salud. La reducción en el empleo total es del $43 \%$, la cual es muy cercana al 40,19\% que manifestaron los empresarios de la Cámara de Comercio de Cali. 
Mora: Análisis del desempleo y la ocupación después de una política estricta de confinamiento...

Tabla 5. Opinión lineal conjunta en torno a la disminución en el empleo por sector

\begin{tabular}{lc}
\hline SECTOR/VARIACIÓN & DISMINUCIÓN \\
\hline AGRICULTURA, GANADERÍA, CAZA, SILVICULTURA y PEZCA & $40,00 \%$ \\
EXPLOTACIÓN DE MINAS Y CANTERAS & $49,70 \%$ \\
INDUSTRIAS MANUFACTURERAS & $39,00 \%$ \\
SUMINISTROS DE ELECTRICIDAD, GAS Y AGUA & $8,00 \%$ \\
CONSTRUCCIÓN & $49,00 \%$ \\
COMERCIO AL POR MAYOR Y AL POR MENOR; REPARACIÓN & $36,70 \%$ \\
DE VEHÍCULOS AUTOMOTORES, MOTOCICLETAS, EFECTOS & \\
PERSONALES Y ENSERES DOMÉSTICOS & \\
HOTELES Y RESTAURANTES & $46,10 \%$ \\
TRANSPORTE, ALMACENAMIENTO Y COMUNICACIONES & $45,30 \%$ \\
INTERMEDIACIÓN FINANCIERA & $25,60 \%$ \\
ACTIVIDADES INMOBILIARIAS, EMPRESARIALES Y DE ALQUILER & $41,20 \%$ \\
ADMINISTRACIÓN PÚBLICA Y DEFENSA; SEGURIDAD SOCIAL DE & $50,00 \%$ \\
AFILIACIÓN OBLIGATORIA & \\
EDUCACIÓN & $49,00 \%$ \\
SERVICIOS SOCIALES Y DE SALUD & $0,00 \%$ \\
OTRAS ACTIVIDADES DE SERVICIOS COMUNITARIOS, SOCIALES & $64,70 \%$ \\
Y PERSONALES & \\
HOGARES PRIVADOS CON SERVICIO DOMÉSTICO & $45,00 \%$ \\
ORGANIZACIONES Y ÓRGANOS EXTRATERRITORIALES & $50,0 \%$ \\
\hline
\end{tabular}

Fuente: elaboración propia a partir de consulta a expertos del ORMET-SIME.

En segundo lugar, se utilizaron series de tiempo, con el fin de pronosticar cual sería el empleo, en los meses siguientes a la pandemia en ausencia de esta.

Se utilizó la serie histórica del empleo desde 2017 hasta el mes de marzo de 2020. La razón por la cual no se escogieron periodos anteriores a 2017 consiste en el cambio en la tendencia tanto en las tasas de ocupación como en la tasa de desempleo de la ciudad de Cali entre el 2011 y el 2016 (DNP, 2020. Cámara de Comercio de Cali, 2019). Y, posteriormente, se eligió marzo de 2020 como periodo final para pronosticar el empleo, ya que la cuarenta 
tiene sus principales afectos posteriores a dicho mes y, por lo tanto, los shocks sobre el mercado laboral empiezan a ocurrir en el mes de abril de 2020.

En primer lugar, se analizaron las de series de tiempo estacional y, a partir del valor del contraste de Hylleberg et al. (1990), que fue de 0,503, se concluye que la serie presenta una raíz unitaria estacional.

De esta forma, se plantea el siguiente modelo en series de tiempo para el pronóstico del empleo:

$$
\left(1-f_{1} L\right)\left(1-F_{1} L^{12}\right)(1-L)\left(1-L^{12}\right) \text { Empleot }_{t}=\epsilon_{t}
$$

Con el fin de determinar el modelo exacto, se eligió el modelo con el menor criterio de información de Akaike (AIC):

Tabla 6. Criterio AIC

\begin{tabular}{lc}
\hline Modelo & AIC \\
\hline$(1,1,0)(1,1,0,12)$ & 298,08 \\
$(1,1,1)(1,1,1,12)$ & 316,07 \\
$(2,1,1)(2,1,1,12)$ & 314,47 \\
$(2,1,0)(2,1,0,12)$ & 329,14 \\
\hline
\end{tabular}

Fuente: elaboración propia a partir de datos de la GEIH (varios años).

De esta forma, la serie del empleo sigue un proceso estacional autorregresivo SARIMA $(1,1,0) \quad(1,1,0,12)$. Una vez definido esto, se pronosticó el empleo desde el mes de abril de 2020 hasta el mes de junio de 2020 .

Considerados estos dos supuestos, se plantearon los siguientes escenarios:

1. La pérdida de empleos es la pronosticada por el ORMET (Tabla 5).

2. La pérdida de empleos ocurre solo en los sectores de comercio (servicios), manufactura (industria), hoteles y restaurantes, transporte y actividades inmobiliarias y en la magnitud prevista por el ORMET mientras que el empleo en los otros sectores no cambia.

3. La pérdida de empleos ocurrió solo en los sectores de comercio (servicios), manufactura (industria), hoteles y restaurantes, transporte y 
Mora: Análisis del desempleo y la ocupación después de una política estricta de confinamiento...

actividades inmobiliarias y su caída fue de un $20 \%$ en estos sectores mientras que el empleo en los otros sectores no cambia.

El escenario 1 es el más pesimista, sin lugar a duda, y tal vez arrojaría el límite inferior en la caída en el empleo.

El escenario 2, se centra en aquellos sectores donde hay consenso, en las diferentes encuestas realizadas en la ciudad, en torno a cuáles serán los más afectados por el COVID-19. Esto no solo se observa de las encuestas, sino que estudios previos realizados por Pinzón et al. (2020) identifican estos sectores para el Valle del Cauca.

El escenario 3, también considera los sectores del escenario 2, pero asume una caída en el empleo del $20 \%$.

Los resultados encontrados se presentan en la Tabla 7.

Tabla 7. Pronósticos de empleo en Cali

\begin{tabular}{ccccccccc}
\hline Mes & $\begin{array}{c}\text { Empleo Pronosticado } \\
\text { (Sin COVID-19) } \\
\text { SARIMA: }(1,1,0)(1,1,0,12)\end{array}$ & $\begin{array}{c}\text { Empleo Real } \\
\text { (COVID-19) }\end{array}$ & Escenario 1 & $\begin{array}{l}\text { Razón de } \\
\text { Predicción }\end{array}$ & Escenario 2 & $\begin{array}{l}\text { Razón de } \\
\text { Predicción }\end{array}$ & Escenario 3 & $\begin{array}{c}\text { Razón de } \\
\text { Predicción }\end{array}$ \\
\hline Abril & 1269927 & 825913 & 824276 & $-0,000861476$ & 960336 & 0,065489 & 1165577 & 0,14961 \\
Mayo & 1317187 & 886445 & 885360 & $-0,000532103$ & 1031503 & 0,065818 & 1183584 & 0,12555 \\
Junio & 1452859 & 980910 & 943013 & $-0,017111675$ & 1098672 & 0,049239 & 1260657 & 0,10897 \\
\hline
\end{tabular}

Escenario 1: Caída en el empleo prevista por expertos del ORMET - Valle del Cauca

Escenario 2: Caída en el empleo solo en sectores principalmente afectados por el COVID-19 y en porcentaje previsto por el ORMET -

Valle del Cauca

Escenario 3: Caída de un $20 \%$ en el empleo solo en sectores principalmente afectados por el COVID-19 según el ORMET - Valle del Cauca

Fuente: elaboración propia.

La Tabla 7, muestra que en el primer escenario se podría generar una pérdida de empleos cercana al $50 \%$. Y, aunque la pérdida es grande, el empleo registrado en el mes de abril y mayo del 2020 utilizando la GEIH muestra que los valores pronosticados son muy cercanos a los valores registrados en los meses de abril, mayo y junio. La diferencia entre los valores reales (columna 
3) y los valores estimados en el escenario 1 (columna 4) es del 0,1\% y esta se va incrementando hasta un $41 \%$ como en el caso del escenario 3 .

Sin embargo, la sola diferencia no dice mucho sobre la capacidad predictiva de la estrategia aquí propuesta para predecir el empleo. Con el fin de analizar dicha capacidad, se utilizará la medida propuesta por Tofallis (2015) o la razón de predicción, la cual consiste en calcular el logaritmo de la razón entre la predicción y el valor real. Se puede observar, que en tanto la razón se encuentre más cercana de cero mejor será la precisión de la predicción. De acuerdo con los valores, el escenario 1 es el que mayor capacidad predictiva muestra mientras que el escenario 3 es el que menor capacidad de predicción tiene. Por otro lado, como es de esperarse, el valor se va alejando a medida que el tiempo va aumentando, ya que se empieza a perder capacidad predictiva del modelo.

La pérdida de empleos en la ciudad de Cali es algo que no solamente concierne a las autoridades municipales, sino también a las autoridades departamentales, debido al peso que tiene el mercado laboral de la ciudad con respecto al mercado laboral departamental como, se puede observar en la Tabla 8.

Tabla 8. Ocupados y desempleados en el Valle del Cauca y Cali

\begin{tabular}{lcccccc}
\hline & \multicolumn{3}{c}{2018} & \multicolumn{3}{c}{2019} \\
\cline { 2 - 7 } & Valle del Cauca & Cali & $\%$ & Valle del Cauca & Cali & $\%$ \\
\hline Ocupados & 2286032 & 1240014 & $54,24 \%$ & 2322715 & 1255227 & $54,04 \%$ \\
Desempleados & 290746 & 161675 & $55,61 \%$ & 313452 & 178786 & $57,04 \%$ \\
\hline
\end{tabular}

Fuente: elaboración propia.

La Tabla 8 muestra el promedio del número de ocupados y desempleados para el 2018 y 2019. De acuerdo con esta tabla, la ciudad de Cali ocupa alrededor del $55 \%$ de la población ocupada del Valle del Cauca y el total de desempleados en la ciudad es alrededor del $55 \%$ del total de desempleados del Valle. 
Mora: Análisis del desempleo y la ocupación después de una política estricta de confinamiento...

\section{El efecto de un shock sobre la tasa de desempleo y ocupación}

Si bien los efectos del COVID-19 son aún impredecibles sobre cualquier economía, ya sea nacional o regional, es posible tratar de aproximar estos efectos en series del mercado laboral como el empleo y el desempleo, a través de la modelación dichas series y el análisis de cuál sería el efecto de un shock sobre dichas series. ${ }^{6}$

Con el fin de modelar el comportamiento de dichas series, se procedió, para comenzar, a contrastar si las series de tasa de ocupación y tasa de desempleo mensual presentaban raíces unitarias estacionales. Los resultados para ambas series corroboran la existencia de raíces unitarias estacionales, lo cual lleva a estimar un modelo SARIMA $(2,1,1)(1,1,0,12)$ para el caso de la tasa de ocupación. ${ }^{7}$ A partir de las anteriores estimaciones se procedió a analizar cuál sería el efecto de un shock en esta serie:

La Figura 1, muestra el efecto de un shock sobre la serie de la tasa de ocupación. Como se puede observar al cabo del mes 10 ya se ha disipado el mismo. El efecto es grande en los primeros 3 meses y se atenúa en el mes 6 . Finalmente, el efecto del shock comienza a desaparecer en el mes 8.

Respecto al desempleo, se modeló la serie de la tasa de desempleo como un SARIMA $(2,1,0)(2,1,0,12) .^{8}$ La Figura 3 muestra los efectos de un shock sobre las series de empleo y desempleo en un periodo de 2 años o 24 meses. Allí, se puede observar los efectos de un shock tardan alrededor de casi 16 meses en desaparecer. Es decir, un impacto sobre el desempleo en Cali, como

6 Las series, tanto de la tasa de ocupación como la tasa de desempleo, comienzan en enero de 2017 hasta febrero de 2020. No se utiliza el mes de marzo ya que en este mes comenzó el shock del COVID-19. Debido a las diferentes medidas tomadas en abril y mayo, con respecto a la reapertura gradual en algunos sectores y el cerramiento en otros como restaurantes y bares, no fue claro incorporar un modelo con cambios estructurales en algún mes ya que no toda la estructura del empleo se ve afectada simétricamente. Por tal razón, se prefirió modelar los efectos del shock a partir de marzo y analizar cuando la serie podría retornar a su valor original.

7 El valor del contraste de Hylleberg et al. (1990) fue de 0,478 para todos los tipos de estacionalidad y se minimizó el AIC con el fin de escoger el modelo definitivo.

8 El valor del contraste de Hylleberg et al. (1990) fue de 0,581 para todos los tipos de estacionalidad y se minimizo el AIC para escoger el modelo definitivo. 
el experimentado a causa del COVID-19, durará más de un año y medio. El efecto, por otro lado, es muy profundo en los dos primeros meses y tiende a suavizarse a partir del décimo mes.

Figura 1. Efectos de un shock sobre la tasa de ocupación

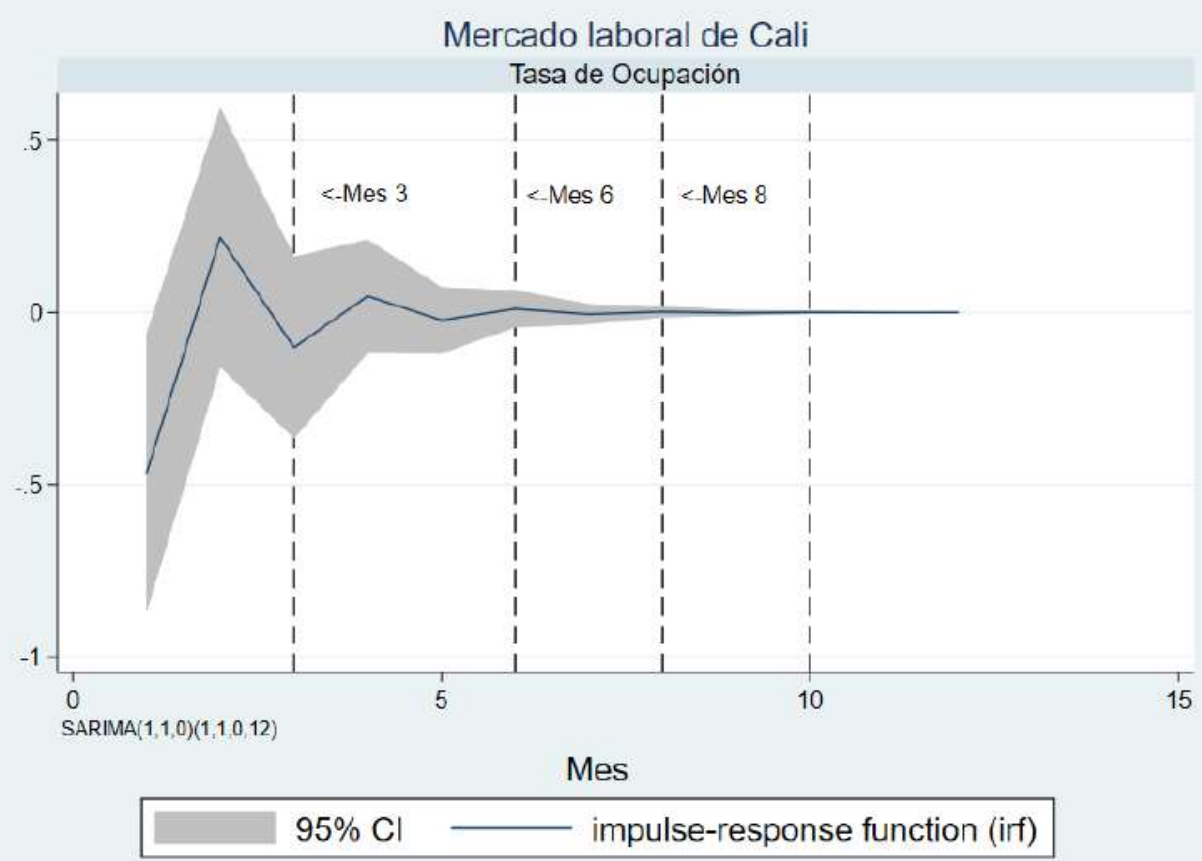

Fuente: elaboración propia a partir de la GEIH (febrero, 2020).

En la Figura 2 se puede observar que los efectos de un shock sobre la tasa de desempleo se prolongan durante más tiempo. Esto es lógico, ya que una vez una persona queda desempleada la probabilidad de conseguir empleo se va reduciendo en la medida en que se dura más periodos de tiempo en situación de desempleo. 
Mora: Análisis del desempleo y la ocupación después de una política estricta de confinamiento...

Figura 2. Shocks sobre la tasa de desempleo

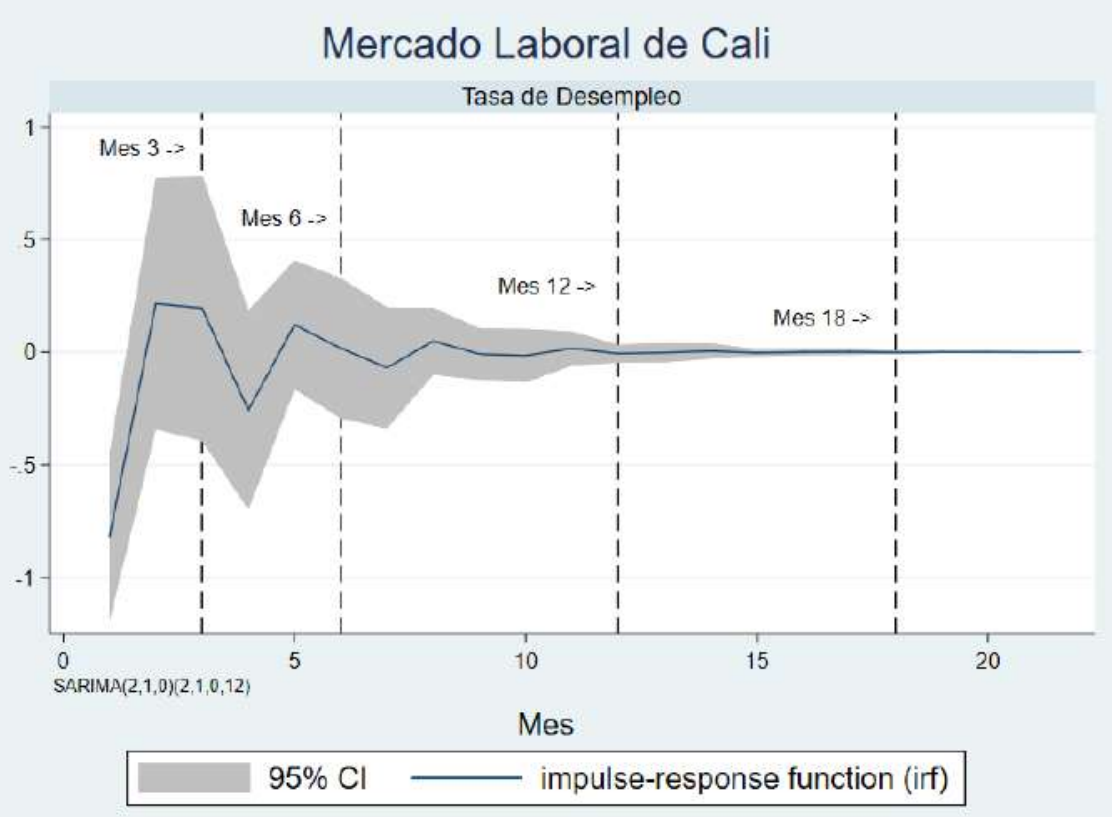

Fuente: elaboración propia a partir de la GEIH (febrero, 2020).

Cabe resaltar que la duración promedio en desempleo para la ciudad de Cali, en el mes de febrero de 2020, fue alrededor de siete meses.

Estas probabilidades no son las mismas para todos los grupos. A continuación, se construyó el estimador no paramétrico Kaplan-Meier (1958) para analizar la salida de una situación de desempleo a una situación de empleo.

La Figura 3a muestra que las mujeres tardan más tiempo a la hora de salir del desempleo al empleo y, al compararla con la Figura 3b se observa que, no solo los jóvenes tienen más barreras a la hora de salir del desempleo, sino que esta situación se agrava para las mujeres jóvenes.

9 Al igual que en el caso de la informalidad, el cambio en la forma de recolección de la GEIH, a partir de marzo de 2020, no permite realizar la gráfica de duración del desempleo en dicho mes y por ello se utilizó el mes de febrero. 
Figura 3. Kaplan-Meier

(Hombres/Mujeres)

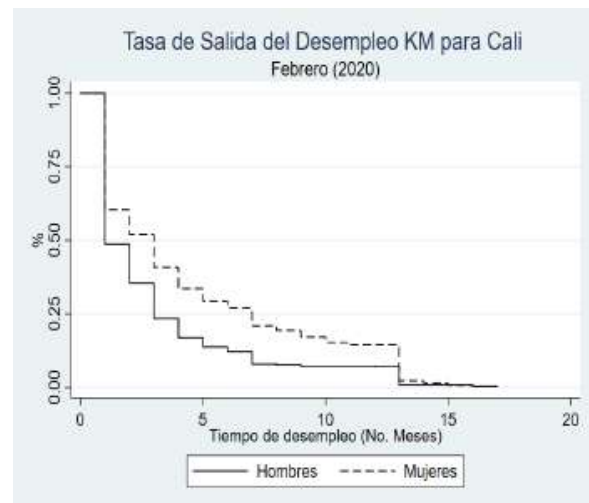

Figura 4. Kaplan-Meier (Jóvenes)

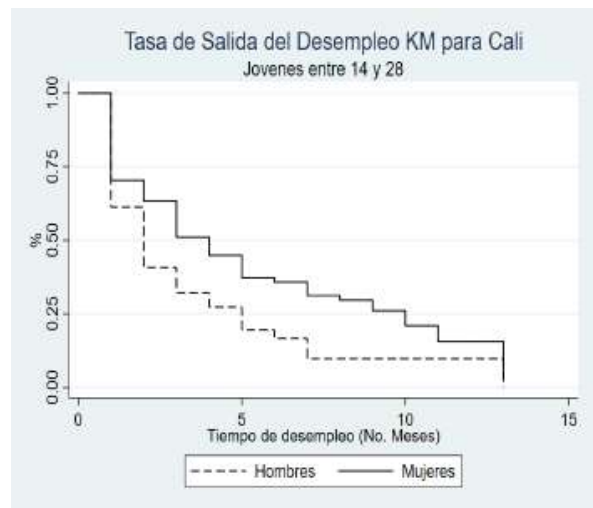

Fuente: elaboración propia a partir de la GEIH (febrero, 2020).

\section{Conclusiones}

El empleo en la ciudad de Cali se concentra en aquellos sectores que más han sido afectados por las medidas tomadas por el gobierno colombiano, para reducir los impactos del COVID-19 sobre la vida y salud de sus ciudadanos. Dichos sectores son el comercio, manufactura, hoteles y restaurantes, transporte y actividades inmobiliarias. Este aspecto, aunado a la alta tasa de informalidad, de un poco más del $50 \%$, que se concentra en los dos primeros estratos, y en las empresas unipersonales, muestra un panorama complejo, donde el COVID-19 ha tenido y tendrá, un gran impacto sobre la generación de empleo y el mercado laboral caleño.

Los estimativos aquí realizados, muestran que en la ciudad se perdieron alrededor de 400000 empleos como efecto del COVID-19 y, además, que los efectos de un shock sobre el desempleo se extenderán hasta el mes dieciocho. En el caso de un shock sobre el empleo los efectos se extenderán alrededor de un año.

A nivel mundial, las políticas activas sobre el empleo se han enfocado, por un lado, en el apoyo a las empresas a través de ayudas a la nómina y, por 
Mora: Análisis del desempleo y la ocupación después de una política estricta de confinamiento...

otro lado, en las ayudas a aquellos trabajadores que caen en el desempleo (por ejemplo, el caso de la ley CARES en Estados Unidos).

Debido al gran efecto que tuvo la pandemia sobre la generación de empleo y, que este se prologará como mínimo durante un año, es importante que la ciudad, a través del mayor endeudamiento aprobado por el consejo municipal, utilice recursos para complementar las ayudas que el gobierno brinda a las empresas (por ejemplo, en programas como el subsidio a la nómina podría incrementar hasta un $20 \%$ adicional de lo que se le da a las empresas) e incluso mantener estas ayudas por un año adicional, cuando el gobierno nacional ya no las brinde.

Con respecto a las ayudas a los trabajadores, que caen en situación de desempleo, estas no se pueden limitar a los primeros tres meses del shock. Los resultados aquí encontrados, muestran que las ayudas se deben reducir de manera escalonada hasta los diez y ocho meses (esto se puede implementar con los recursos adicionales aprobados por el consejo municipal para combatir los efectos de la pandemia). Por otro lado, debido al tamaño de la informalidad en Cali, las ayudas que ya estableció el gobierno de $\$ 160000$ por hogar (para aquellos registrados en las bases del SISBEN) deben acompañarse con otras medidas que incentiven la formalización y reduzcan la transición entre formalidad e informalidad, ya que estas ayudas no son suficientes dada la magnitud de esta en la ciudad. Es muy importante tener en cuenta lo anterior, si no se quiere que la informalidad supere la barrera del $60 \%$. Los despidos que ha ocasionado y seguirá ocasionando el COVID-19 llevarán a que la informalidad en Cali se incremente, sin duda alguna. Un seguro de desempleo como el existente en España amortiguaría esta transición hacia la informalidad; sin embargo, esta es una estrategia que se debe implementar a nivel nacional. ${ }^{10}$

10 En España, dependiendo de las condiciones, el trabajador recibe el $70 \%$ de la base cotización en el empleo anterior, y del séptimo mes en adelante, del $50 \%$ de la base cotización. Esto permite que el tránsito de una situación de desempleo hacia la informalidad se reduzca. En Colombia, la probabilidad de que estando en condición de formalidad en el periodo $(t-1)$ se pase a una situación de informalidad en $t$ es del $49 \%$, mientras que si el trabajador se encuentra en condición de informalidad en el periodo $t-1$ la probabilidad de que pase a una situación de formalidad en $t$ es del $36 \%$ (Mora y Muro, pp. 66, 2017). 


\section{Agradecimientos}

Agradezco los comentarios a una primera versión de este documento, la cual circuló como: El efecto del Coronavirus (COVID-19) sobre el mercado laboral en la ciudad de Cali. Icesi Working Papers, No. 11 (2020). También agradezco los comentarios de los dos evaluadores y del editor de la revista. Los errores que persisten son de mi absoluta responsabilidad

\section{Referencias}

Alfaro, L., Álvarez, A., Becerra, O., Eslava, M., Isaacs, M. \& Urdaneta, N. (2020). El empleo y los cierres por COVID19. Observatorio de coyuntura económica y social, Universidad de los Andes, Facultad de Economía. https:/economia.uniandes.edu.co/centros-de-investigacion/ cede/observatorio-de-coyuntura-economica-y-social

Alstadsæter, A., Bratsberg, B., Eielsen, G., Kopczuk, W., Markussen, S., Raaum, O. \& Røed, K. (2020). The First Weeks of the Coronavirus Crisis: Who Got Hit, When and Why? Evidence from Norway. National Bureau of Economic Research. https://doi.org/10.3386/w27131

Angelucci, M., Angrisani, M., Bennett, D., Kapteyn, A. \& Schaner, S. (2020). Remote Work and the Heterogeneous Impact of COVID-19 on Employment and Health. National Bureau of Economic Research. https://doi.org/10.3386/w27749

Bartik, A., Bertrand, M., Lin, F., Rothstein, J. \& Unrath, M. (2020). Measuring the labor market at the onset of the COVID-19 crisis. National Bureau of Economic Research. https://doi.org/10.3386/w2 7613

Boar, C. \& Mongey, S. (2020). Dynamic Trade-offs and Labor Supply Under the CARES Act. National Bureau of Economic Research. https://doi.or $\mathrm{g} / 10.3386 / \mathrm{w} 27727$

Botero, J. y Montañez, D. (2020). Efectos en la economía colombiana del COVID-19: Un análisis de equilibrio general computable. Economía 
Mora: Análisis del desempleo y la ocupación después de una política estricta de confinamiento...

colombiana: Análisis de coyuntura. Universidad EAFIT. https://www. eafit.edu.co/escuelas/economiayfinanzas/cief/Documents/informe-esp ecial-2020-abril.pdf

Bonet, J., Ricciulli, D., Pérez, G., Galvis, L., Haddad, E., Araújo, I. y Perobelli, F. (2020). Impacto económico regional del COVID-19 en Colombia: un análisis insumo-producto. Documentos de trabajo sobre economía regional y urbana, 288. https://repositorio.banrep.gov.co/bitstream/ha ndle/20.500.12134/9843/DTSERU_288.pdf

Cámara de Comercio de Cali. (2019). Ritmo laboral Grupo Estudios Empresariales y de competitividad. https://www.ccc.org.co/wp-cont ent/uploads/2019/12/Ritmo-Laboral-N59.pdf

Coibion, O., Gorodnichenko, Y. \& Weber, M. (2020). Labor Markets During the COVID-19 Crisis: A Preliminary View. National Bureau of Economic Research. https://doi.org/10.3386/w27017

Clemen, R. T. \& Winkler, R. L. (1987). Calibrating and Combining Precipitation Probability Forecasts. En Probability and Bayesian Statistics (pp. 97-110). Springer US. https://doi.org/10.1007/978-1-4613-18859_10

Dingel, J. \& Neiman, B. (2020). How Many Jobs Can be Done at Home? National Bureau of Economic Research. https://doi.org/10.3386/w269 48

DNP (2017). Mercado laboral urbano. Resultados 2017: Cali. Dirección de Estudios Económicos. https://colaboracion.dnp.gov.co/CDT/Estudios \%20Econmicos/5.Informe\%20MLU\%20Cali\%202017.pdf

Eslava, M. \& Isaacs, M. (2020). Vulnerabilidad del empleo a la emergencia de COVID19 en Colombia. Observatorio de coyuntura económica y social, Universidad de los Andes, Facultad de Economía. https:/economia.uni andes.edu.co/images/archivos/pdfs/CEDE/Observatorio-coyuntura/Gr \%C3\%A1ficos_Empleos_-_Nacional-07042020_-_1.pdf 
Fernández, C. (2020). Impacto en el mercado laboral de las medidas de aislamiento para combatir el COVID-19. Fedesarrollo. https://www.re pository.fedesarrollo.org.co/handle/11445/3935

Forsythe, E., Kahn, L., Lange, F. \& Wiczer, D. (2020). Labor Demand in the time of COVID-19: Evidence from vacancy postings and UI claims. National Bureau of Economic Research. https://doi.org/10.3386/w270 61

Genest, C. \& McConway, K. J. (1990). Allocating the weights in the linear opinion pool. Journal of Forecasting, 9(1), 53-73. https://doi.org/10.100 2/for.3980090106

Hylleberg, S., Engle, R. F., Granger, C. W. J. \& Yoo, B. S. (1990). Seasonal integration and cointegration. Journal of Econometrics, 44(1-2), 215-238. https://doi.org/10.1016/0304-4076(90)90080-d

Jaramillo, I. D., Londoño, D., Rodríguez, P. \& García, A. (2020). La vulnerabilidad del mercado laboral colombiano al COVID-19. Observatorio Laboral LABOUR. https://017035e1-a1be-4007-a4b 9-2f9be5a00e35.filesusr.com/ugd/c80f3a_edc97b6b3b9c43e088bb8ab 2c39fbb38.pdf

Kaplan, E. L. \& Meier, P. (1958). Nonparametric Estimation from Incomplete Observations. Journal of the American Statistical Association, 53(282), 457-481. https://doi.org/10.1080/01621459.1958.10501452

Mora, J. J. (2017). La informalidad laboral colombiana en los últimos años: análisis y perspectivas de política pública. Revista de Métodos Cuantitativos para la Economía y la Empresa, 24(1), 89-128.

Mora J. J. \& Caicedo, C. (2018). Prospectiva laboral en el sector de química, farmacéutica, nutracéutica y cosméditca en el Valle del Cauca. Icesi Working Papers No. 10. https://www.icesi.edu.co/images/departam entos/FCAE/economia/apuntesEconomia/IEWP\%202018-10.pdf

Mora, J. J. \& Muro, J. (2017). Dynamic Effects of the Minimum Wage on Informality in Colombia. LABOUR, 31(1), 59-72. https://doi.org/10.1 111/labr.12087 
Mora: Análisis del desempleo y la ocupación después de una política estricta de confinamiento...

Morales, L. F., Bonilla-Mejía, L., Pulido, J., Pulido-Mahecha, K. L., Hermida, D., Flórez, L. A. \& Lasso-Valderrama, F. (2020). Effects of the COVID19 Pandemic on the Colombian Labor Market: Disentangling the Effect of Sector-Specific Mobility Restrictions. Banco de la República de Colombia. https://doi.org/10.32468/be.1129

Pinzón, L. F., Mena, S. V., Ruiz, C. H. \& Restrepo, J.C. (2020). Aproximación a los impactos de la pandemia del COVID-19 en el Valle del Cauca. Estudios Socioeconómicos 32. https://www.valledelcauca.gov.co/loader. php?IServicio $=$ Tools $2 \& l$ Tipo $=$ viewpdf\&id $=42221$

Seaver, D. A. (1978). Assessing probability with multiple individuals: Group interaction versus mathematical aggregation (Report No. 78-3). Social Science Research Institute, University of Southern California.

Serna, H. M., Barrera, A. \& Castro, E. S. (2020). Índice de riesgo a la pérdida del empleo en Colombia durante la coyuntura del COVID-19. Manizales: ORMET, Universidad de Manizales.

Tofallis, C. (2015). A better measure of relative prediction accuracy for model selection and model estimation. Journal of the Operational Research Society, 66(8), 1352-1362. https://doi.org/10.1057/jors.2014.103

Qiu, Y., Chen X. \& Shi, W. (2020). Impacts of social and economic factors on the transmission of coronavirus disease 2019 (COVID-19) in China. Journal of Population Economics 33, 1127-1172 https://doi.org/10.100 7/s00148-020-00778-2 